\title{
ON THE CENTRAL LIMIT THEOREM FOR DYNAMICAL SYSTEMS
}

\author{
ROBERT BURTON AND MANFRED DENKER
}

\begin{abstract}
Given an aperiodic dynamical system $(X, T, \mu)$ then there is an $f \in L^{2}(\mu)$ with $\int f d \mu=0$ satisfying the Central Limit Theorem, i.e. if $S_{m} f=$ $f+f \circ T+\cdots+f \circ T^{m-1}$ and $\sigma_{m}=\left\|S_{m} f\right\|_{2}$ then$$
\mu\left\{x \mid \frac{S_{m} f(x)}{\sigma_{m}}<u\right\} \rightarrow(2 \pi)^{-1 / 2} \int_{-\infty}^{u} \exp \left[\frac{-v^{2}}{2}\right] d v
$$

The analogous result also holds for flows.

I. Introduction. The most important and most studied theorem in probability theory is the Central Limit Theorem (CLT) which may be stated in the context of dynamical systems. If $(X, T, \mu)$ is a dynamical system (i.e. $T$ is a measurable, measure-preserving transformation of the Lebesgue probability space $(X, \mu))$ and if $f \in L^{2}(\mu)$ is centered, i.e. $\int_{X} f d \mu=0$, with $f, f \circ T, f \circ T^{2}, \ldots$ forming an independent sequence then

$$
\mu\left\{x \in X \mid \frac{S_{m} f(x)}{\left\|S_{m} f\right\|}<u\right\} \rightarrow \Phi(u) \quad \text { as } m \rightarrow \infty
$$

where $S_{m} f=f+f \circ T+\cdots+f \circ T^{m-1},\left\|S_{m} f\right\|=L^{2}$-norm of $S_{m} f=$ standard deviation of $S_{m} f$, and $\Phi(u)=2(\pi)^{-1 / 2} \int_{-\infty}^{u} \exp \left[-v^{2} / 2\right] d v$. In this case $\left\|S_{m} f\right\|=$ $\sqrt{m\|f\|}$ but we shall drop the independent assumption above, and we shall then say that $f \in L^{2}(\mu)$ satisfies the CLT if (1) holds.

Many generalizations of the CLT are in the literature where (1) is shown to hold for a wider class of functions. To the authors' knowledge, all of these work by weakening the independence assumptions. As examples, (1) still holds for certain functions $f$ where $f, f \circ T, \ldots$ satisfy mixing conditions of Rosenblatt or Ibragimov $[\mathbf{8}, \mathbf{1 4}]$ or form a martingale [2] or satisfy positive dependence conditions with summable convariance functions as in Newman and Wright [10].

Some attention has been paid to CLT for special dynamical systems, such as Ratner [12], Denker and Philipp [4], Hofbauer and Keller [7], and others cited there. All these results are deduced from kinds of mixing as described above. Similar things can be said about flows, built under a function, but the situation is somewhat more complicated. All these theorems and examples imply at least the $K$-property, so far no CLT for a dynamical system seems to be published where

Received by the editors September 5, 1985 and, in revised form, July 28, 1986.

1980 Mathematics Subject Classification (1985 Revision). Primary 60F05; Secondary 28D05.

Key words and phrases. Central limit theorem, dynamical systems.

The first author was supported in part by the Alexander von Humboldt Foundation and NSF grant \#MCS-800 5172. 
the system is not $K$, especially where the system has zero entropy. On the other hand examples of zero entropy Gaussian processes are known to exist, e.g. [9]. The question whether the irrational rotation has a function with CLT was raised by J. P. Conze (see [3, p. 159]). See also the forthcoming paper of Dürr and Goldstein [11]. To make the idea of this paper clear, we would like to point out that there are other types of CLTs known in probability and number theory, and we would like to make them applicable to dynamical systems, as was done for the probabilistic mixing CLTs.

In particular, another sort of generalization of the CLT due to Salem and Zygmund [16] uses the fact that trigonometric functions whose frequencies are far enough apart are 'almost independent'. Specifically given a lacunary sequence satisfying $n_{k+1}>3 n_{k}$ for $k=1,2, \ldots$ and $C_{k}, D_{k} \in \mathbf{R}$ with $a_{k}=\sqrt{\left(C_{k}^{2}+D_{k}^{2}\right)}>0$ then

$$
\begin{aligned}
\mu\left\{x \in[0,1] \mid \frac{1}{A_{m}} \sum_{k=1}^{m} C_{k} \cos \left(2 \pi n_{k} x\right)+D_{k} \sin \left(2 \pi n_{k} x\right)<u\right\} & \rightarrow \Phi(u) \\
& \text { as } m \rightarrow \infty
\end{aligned}
$$

where $A_{m}=\left\{\frac{1}{2}\left(C_{1}^{2}+D_{1}^{2}+\cdots+C_{m}^{2}+D_{m}^{2}\right)\right\}^{1 / 2}$ provided $a_{m} / A_{m} \rightarrow 0$ and $A_{m} \rightarrow \infty$ and where $\mu$ denotes Lebesgue measures.

Rothstein [15] has constructed a class of Vershik processes of zero entropy and a function $f$ taking only a finite number of values with $\left\|S_{m} f\right\|^{2} / m \rightarrow 1$ that satisfies the CLT.

The main result of the present paper is

THEOREM 1. If $(X, T, \mu)$ is an aperiodic dynamical system then there exists $f \in L^{2}(\mu)$ which satisfies the $C L T$.

In the second part, we prove Theorem 1 in the case where $(X, T, \mu)$ is an irrational rotation using Fourier series. In the third part, Rochlin towers will provide a structure that will allow us to imitate the construction for a rotation in the general case.

REMARK. If the entropy of $(X, T, \mu)$ is positive, then by Sinai's theorem, there are Bernoulli factors so Theorem 1 is trivial. However, the method of construction will be flexible enough to obtain the following results.

THEOREM 2. The function $f$ in Theorem 1 may be chosen to be a generator for the $\sigma$-field.

We may also extend the theorem to flows built under a function by making the construction on the base transformation and appealing to standard arguments, see Rényi [13, p. 390].

THEOREM 3. Let $T_{t}(t \in \mathbf{R})$ be an aperiodic measure preserving flow on $(X, \mu)$. Then there exists a function $f \in L^{2}(\mu)$ satisfying the CLT, i.e. letting $S_{t} f=$ $\int_{0}^{t} f \circ T_{\tau} d \tau ;$ then

$$
\mu\left\{x \mid S_{t} f(x) /\left\|S_{t} f\right\| \leq u\right\} \rightarrow \Phi(u) .
$$

We conjecture that there are such functions $f$ satisfying the almost sure invariance principle, namely there is a time change $A(t)$ and possibly after enlarging the 
probability space, a Brownian motion $B(t)$ so that $\left|S_{A(t)} f-B(t)\right|=O\left(t^{1 / 2-\lambda}\right)$ for some $\lambda>0$. See Philipp and Stout $[\mathbf{1 1}$, p. $60 \mathrm{ff}$.$] .$

REMARK. It is an open problem to determine the category in $L^{2}$ of the set of functions satisfying the CLT. It will be clear from the proofs that this set of functions is dense in $L^{2}$, if $\mu$ is ergodic. This also follows more directly from the observation that the subspace $\left\{\varphi \circ T-\varphi: \varphi \in L^{2}(\mu)\right\}$ is dense in $\left\{g \in L^{2}(\mu): \int g d \mu=0\right\}$ (see Gordin and Lifsic [6, Remark 1, p. 393] and Bhattacharya [1, Proposition 2.2, p. 191]. We would like to thank the referee for helpful suggestions, especially for pointing out this last fact.

II. CLT for rotations. Now let $X=\mathrm{R} / Z$ be the unit circle with $\mu$ Lebesgue measure and suppose $\alpha \in X$ is irrational and $T: X \rightarrow X$ is defined by $T(x)=x+\alpha$. Now any $f \in L^{2}(\mu)$ with $\int_{X} f d \mu=0$ has a Fourier representation $f=\sum_{k=-\infty}^{\infty} b_{k} g_{k}$ where $b_{0}=0$ and $g_{k}(x)=e^{2 \pi i k x}$ are eigenfunctions. Our functions will be real valued with real Fourier coefficients so $b_{k}=b_{-k}$ and $f(x)=\sum_{k=1}^{\infty} 2 b_{k} \cos (2 \pi k x)$. Now $T g_{k}=\beta^{k} g_{k}$ where $\beta=e^{2 \pi i \alpha}$ and it follows easily that

$$
S_{m} f=\sum_{k=-\infty}^{\infty} b_{k} \frac{1-\beta^{m k}}{1-\beta^{k}} g_{k}
$$

and

$$
\sigma_{m}^{2}:=\left\|S_{m} f\right\|^{2}=\sum_{k=1}^{\infty} 2 b_{k}^{2}\left|\frac{1-\beta^{m k}}{1-\beta^{k}}\right|^{2} .
$$

The examples we construct will all have the following structure: there are $J_{1}, J_{2}, \ldots$ subsets of the natural numbers so $k_{n}=\#\left(J_{n}\right), \min \left(J_{n+1}\right)>3 \max \left(J_{n}\right)$ and $i, j \in J_{n}, i<j \Rightarrow 3 i<j$ for each $n=1,2, \ldots$.

We also have real numbers $\varepsilon_{n} \downarrow 0, \bar{\varepsilon}_{n} \downarrow 0$ such that $j \in J_{n}$ implies $\beta^{j}$ is in the first quadrant of the circle and $\varepsilon_{n}>\left|1-\beta^{j}\right|>\varepsilon_{n}-\bar{\varepsilon}_{n}>\varepsilon / 2$ which gives for $j \in J_{n}$, and $m \varepsilon_{n} \leq 1$

$$
m>\left|\frac{1-\beta^{j m}}{1-\beta^{j}}\right|>m\left(1-m \varepsilon_{n}\right) \geq 0 .
$$

Further, we have numbers $a_{n}>0$ for $n=1,2, \ldots$ so $j \in J_{n} \Rightarrow b_{j}=b_{-j}=a_{n}$ and $j \notin \bigcup_{n} J_{n} \Rightarrow b_{j}=0 . f$ so defined will belong to $L^{2}$ provided

$$
\sum_{n=1}^{\infty} a_{n}^{2} k_{n}<\infty
$$

and because $\alpha$ is irrational, given $\varepsilon_{n}, \bar{\varepsilon}, k_{n}, a_{n}$ satisfying the above conditions, we can find $J_{n}$ as above and define $f$.

For such an $f$ we can rewrite (4)

$$
\left\|S_{m} f\right\|^{2}=\sum_{n=1}^{\infty} 2 a_{n}^{2} \sum_{j \in J_{n}}\left|\frac{1-\beta^{j m}}{1-\beta^{j}}\right|^{2} .
$$

Now we set $L(n)=2 a_{n}^{2} k_{n}$. Because $4\left|1-\beta^{j}\right|^{-2} \geq\left|\left(1-\beta^{j m}\right) /\left(1-\beta^{j}\right)\right|^{2}$ and (5) we have the following estimate on the variance of $S_{m} f$ : for any $n_{0}$ such that 
$m \varepsilon_{n_{0}} \leq 1$

$$
\begin{aligned}
L\left(n_{0}\right) m^{2}\left(1-m \varepsilon_{n_{0}}\right)^{2} & \leq\left\|S_{m} f\right\|^{2} \\
& \leq \sum_{n<n_{0}} 16 L(n) \varepsilon_{n}^{-2}+L\left(n_{0}\right) m^{2}+m^{2} \sum_{n>n_{0}} L(n) .
\end{aligned}
$$

LEMMA 1. For each $\gamma, 0<\gamma<2$, there is an $f$ of the above form and a subsequence $N_{0} \subseteq \mathbf{N}$ so that for $m \in N_{0}$

$$
\left\|S_{m} f\right\|^{2} \sim m^{2-\gamma}
$$

and (1) is satisfied, i.e. $S_{m} f$ satisfies the CLT along the subsequence $N_{0}$.

PROOF. Set $L(n)=2^{-\gamma n^{2}}, m=2^{n_{0}^{2}}$, and $\varepsilon_{n}=2^{-n^{2}-n}$. Then for large enough $n_{0}(8)$ becomes

$$
\begin{aligned}
m^{-\gamma} m^{2}\left(1-2^{-n_{0}}\right) & \leq\left\|S_{m} f\right\|^{2} \\
& \leq 4 \sum_{n<n_{0}}\left(2^{-\gamma n^{2}}\right)\left(2^{2 n^{2}+2 n}\right)+m^{2-\gamma}+m^{2} \sum_{n>n_{0}} 2^{-\gamma n^{2}} \\
& \leq 8\left(2^{(2-\gamma)\left(n_{0}-1\right)^{2}}\right)+m^{2-\gamma}+2 m^{2} 2^{-\gamma\left(n_{0}+1\right)^{2}}
\end{aligned}
$$

which implies (9) for $N_{0}=\left\{2^{n^{2}} \mid n=1,2, \ldots\right\}$.

This estimate on the variance of $S_{m} f$ shows that $S_{m} f$ is well approximated in $L^{2}$ by the terms in its Fourier series coming from the block corresponding to $J_{n_{0}}$. Thus to prove (1) for $m=2^{n_{0}^{2}} \rightarrow \infty$ we may replace $S_{m} f$ by

$$
a_{n_{0}} \sum_{ \pm j \in J_{n_{0}}}\left(\frac{1-\beta^{j m}}{1-\beta^{j}}\right) g_{j} .
$$

Knowing $k_{n_{0}}$ we may assume $\bar{\varepsilon}_{n_{0}}$ is so small that $\left|1-\beta^{j}\right|$ is essentially $\varepsilon_{n_{0}}$ and so by making another small $L^{2}$ error, we may replace

$$
\frac{1-\beta^{j m}}{1-\beta^{j}} g_{j}+\frac{1-\beta^{-j m}}{1-\beta^{-j}} g_{j}
$$

by

$$
C_{n_{0}} \cos (2 \pi j x)+D_{n_{0}} \sin (2 \pi j x)
$$

where $C_{n_{0}}, D_{n_{0}}$ depend only on $\varepsilon_{n_{0}}$.

Thus the lemma will be proven if we can satisfy (1) with $S_{m} f /\left\|S_{m} f\right\|$ replaced by

$$
\frac{1}{A_{n_{0}} \sqrt{k_{n_{0}}}} \sum_{j \in J_{n_{0}}}\left(C_{n_{0}} \cos (2 \pi j x)+D_{n_{0}} \sin (2 \pi j x)\right)
$$

where $A_{n_{0}}=\sqrt{\left(C_{n_{0}}^{2}+D_{n_{0}}^{2}\right)} / 2$. But this is clear because we are still free to make $k_{n_{0}}$ as large as we want which makes this expression arbitrarily close in distribution to the standard normal as is written in (2).

THEOREM 1a. In the case where $(X, T, \mu)$ is an irrational rotation, there exist $f \in L^{2}$ satisfying the $C L T$.

PROOF. We will slightly modify the construction in the proof of Lemma 1. For large $m$ let $n_{0}=n_{0}(m)=\sup \left\{n \mid 2^{n^{2}} \leq m\right\}$. The estimates above show that most of the variance of $S_{m} f$ is carried by terms from the $J_{n_{0}}$ and $J_{n_{0}+1}$ blocks. 
Thus

$$
\frac{S_{m} f(x)}{\left\|S_{m} f\right\|}=\left\|S_{m} f\right\|^{-1} \sum_{k=1}^{\infty} 2 a_{k} \sum_{ \pm j \in J_{k}} \frac{1-\beta^{m j}}{1-\beta^{j}} \cos 2 \pi j x \quad(m \geq 1)
$$

is $L^{2}$-equivalent to

$$
\begin{gathered}
\left(2 a_{n_{0}}^{2} \sum_{j \in J_{n_{0}}}\left|\frac{1-\beta^{j m}}{1-\beta^{j}}\right|^{2}+2 a_{n_{0}+1} \sum_{j \in J_{n_{0}+1}}\left|\frac{1-\beta^{j m}}{1-\beta^{j}}\right|^{2}\right)^{-1} \\
\cdot\left(a_{n_{0}} \sum_{ \pm j \in J_{n_{0}}} \frac{1-\beta^{j m}}{1-\beta^{j}} g_{j}+a_{n_{0}+1} \sum_{ \pm j \in J_{n_{0}+1}} \frac{1-\beta^{j m}}{1-\beta^{j}} g_{j}\right)
\end{gathered}
$$

as $m \rightarrow \infty$, i.e. both functions will have the same limit distribution if there is any. We still are free to choose the size $k_{n}$ of the sets $J_{n}$ as large as we want. If $n$ is fixed choose $k_{n}$ so large that according to (2) for each $2^{n^{2}} \leq m<2^{(n+1)^{2}}$

$$
A_{m}^{-1}\left(a_{n} \sum_{ \pm j \in J_{n}} \frac{1-\beta^{j m}}{1-\beta^{j}} g_{j}+a_{n+1} \sum_{ \pm j \in J_{n+1}} \frac{1-\beta^{j m}}{1-\beta^{j}} g_{j}\right)
$$

and for each $2^{(n-1)^{2}} \leq m<2^{n^{2}}$

$$
A_{m}^{-1}\left(a_{n-1} \sum_{ \pm j \in J_{n-1}} \frac{1-\beta^{j m}}{1-\beta^{j}} g_{j}+a_{n} \sum_{ \pm j \in J_{n}} \frac{1-\beta^{j m}}{1-\beta^{j}} g_{j}\right)
$$

are approaching the standard normal distribution uniformly in $m$ as $n \rightarrow \infty$. Here,

$$
\begin{aligned}
A_{m}^{2}= & \frac{1}{2}\left(a_{n}^{2} \sum_{j \in J_{n}}\left\{\left(2 \operatorname{Re} \frac{1-\beta^{j m}}{1-\beta^{j}}\right)^{2}+\left(2 \operatorname{Im} \frac{1-\beta^{j m}}{1-\beta^{j}}\right)^{2}\right\}\right) \\
& +a_{n \pm 1}^{2} \sum_{j \in J_{n \pm 1}}\left\{\left(2 \operatorname{Re} \frac{1-\beta^{j m}}{1-\beta^{j}}\right)^{2}+\left(2 \operatorname{Im} \frac{1-\beta^{j m}}{1-\beta^{j}}\right)^{2}\right\} \\
= & 2 a_{n}^{2} \sum_{j \in J_{n}}\left|\frac{1-\beta^{j m}}{1-\beta^{j}}\right|^{2}+2 a_{n \pm 1}^{2} \sum_{j \in J_{n \pm 1}}\left|\frac{1-\beta^{j m}}{1-\beta^{j}}\right|^{2}
\end{aligned}
$$

and consequently (10) is asymptotically normal. This proves the theorem.

III. Proof of Theorem 1. Let $(X, T, \mu)$ be an arbitrary aperiodic dynamical system, invertible or not. We will use a structure of Rochlin towers as a frame that will allow us to imitate the construction for rotations. Recall that a measurable set $F \subset X$ is called an $(N, \varepsilon)$-Rochlin set if $F, T^{-1} F, \ldots, T^{-N+1} F$ are disjoint and $\mu\left(\bigcup_{l=0}^{N-1} T^{-l} F\right)>1-\varepsilon$. Rochlin's lemma states that these sets exist for any $\varepsilon>0$ and $N \in \mathrm{N}$, provided the system is aperiodic.

Definition. Let $K, N, L$ be integers, $K<N, K$ odd, $N$ even and let $\varepsilon>0$. If $F$ is an $(N L, \varepsilon)$-Rochlin set, a function $g: X \rightarrow\{-1,0,1\}$ will be called special for $(F, N, K)$ if it has the following properties:

(i) $g(x)=0 \Leftrightarrow x \notin \bigcup_{l=0}^{N L-1} T^{-l} F$. 
(ii) $\mu(\{x \in F \mid g(x)=1\})=\mu(\{x \in F \mid g(x)=-1\})=\frac{1}{2} \mu(F)$.

(iii) There exist functions $g^{l}: F \rightarrow\{-1,1\}(l=0,1, \ldots, N-1)$ such that $g^{0}, g^{2}$, $g^{4}, \ldots, g^{N-2}$ are independent, identically distributed with respect to the measure $\left.\mu\right|_{F}$ and such that $g(x)=g^{l}\left(T^{l} x\right)$ if $x \in T^{-l} F(l=0, \ldots, N-1)$.

(iv) $g^{2 l}(x)=-g^{2 l+K}(x)$ where indices are $\bmod N$.

(v) If $x \in T^{-j N+1} F(j=2,3, \ldots, L)$ and if $0 \leq l<N$, then $g\left(T^{l}(x)\right)=$ $g\left(T^{N+l}(x)\right)$.

Before we proceed with a construction of special functions, let us note the following properties, which immediately follow from the definition.

LEMMA 2. Let $g$ be a special function for $(F, N, K)$. Then we have

(1) $\int_{X} g d \mu=0$.

(2) If $x \in T^{-j N+1} F$ for some $j=1,2, \ldots, L$, then $\sum_{l=0}^{N-1} g\left(T^{l}(x)\right)=0$.

(3) Let $K \leq m<N-K$ and $0 \leq l<N$. Then there exists a set $J \subset$ $\{0,1, \ldots, m-1\}$ of cardinality $K-1, K$ or $K+1$ such that for every

$$
x \in \bigcup_{j=1}^{L} T^{-j N+1} F
$$

we have

$$
\sum_{i=0}^{m-1} g\left(T^{i+l}(x)\right)=\sum_{i \in J} g\left(T^{i+l}(x)\right) .
$$

More precisely, if $l$ and $m+l-1$ are even then

$$
J=\{1,3, \ldots, K, m-K, m-K+2, \ldots, m-1\} .
$$

If $l$ is even and $m+l-1$ is odd, then

$$
J+\{1,3, \ldots, K, m-K+1, m-K+3, \ldots, m-2\} .
$$

If $l$ is odd and $m+l-1$ is even then

$$
J+\{0,2, \ldots, K-1, m-K, m-K+2, \ldots, m-1\} .
$$

If $l$ and $m+l-1$ are odd, then

$$
J=\{0,2, \ldots, K-1, m-K+1, m-K+3, \ldots, m-2\} .
$$

LEMMA 3. Let $g$ be a special function for $(F, N, K)$. Then we have

(1) $\left|S_{m} g(x)\right| \leq 2(K+1), m \geq 0, x \in X$, where $S_{m} g(x)=\sum_{l=0}^{m-1} g\left(T^{l}(x)\right)$.

(2) If $0 \leq m \leq K$ then $(1-\delta) m \leq\left\|S_{m} g\right\|^{2} \leq(1+\delta) m$ and

$$
\mu\left(\left\{x \in X \mid S_{m} g(x) / \sqrt{m} \leq t\right\}\right)=\Phi(t)+\varsigma+O\left(m^{-1 / 2}\right)
$$

where $\max (\delta, \varsigma) \rightarrow 0$ as $m\left(L^{-1}+\varepsilon\right) \rightarrow 0, m, L \rightarrow \infty$ and $\varepsilon \rightarrow 0$.

(3) If $K \leq m<N-K$ then $(1-\delta) K \leq\left\|S_{m} g\right\|^{2} \leq(1+\delta) K$ and

$$
\mu\left(\left\{x \in X \mid S_{m} g(x) / \sqrt{K} \leq t\right\}\right)=\Phi(t)+\varsigma+O\left(K^{-1 / 2}\right)
$$

where $\max (\delta, \varsigma) \rightarrow 0$ as $K\left(L^{-1}+\varepsilon\right) \rightarrow 0, K, L \rightarrow \infty$ and $\varepsilon \rightarrow 0$. 
ProOF. (1) follows immediately from Lemma 2(2), (3) and (i) in the definition of a special function. (2) is similar to (3), hence we only prove (3). Let $G=$ $\bigcup_{l=N}^{L N-1} T^{-l} F$ and $B=G^{c}$. Then $\mu(G)=(L-1) N \mu(F) \geq(L-1) N(1-\varepsilon) / L N=$ $1-\varepsilon(L-1) / L-1 / L$. We have

$$
\begin{gathered}
\left\|S_{m} g\right\|^{2}=\int_{G}\left|S_{m} g\right|^{2} d \mu+\int_{B}\left|S_{m} g\right|^{2} d \mu \\
\int_{B}\left|S_{m} g\right|^{2} d \mu \leq 4(K+1)^{2} \mu(B) \leq 4(K+1)^{2}\left(\varepsilon+L^{-1}\right)
\end{gathered}
$$

and

$$
\int_{G}\left|S_{m} g\right|^{2} d \mu=\sum_{l=N}^{L N-1} \int_{T^{-l} F}\left|S_{m} g\right|^{2} d \mu .
$$

Fix $l$ and denote by $J$ the set of at most $K+1$ indices determined in Lemma 2(3) for $m$ and $j N-1-l$, where $l=j N-1-l^{\prime}, 0 \leq l^{\prime}<N, j \geq 2$. Let $x \in T^{-l} F$. Choose $y \in T^{-j N+1} F$ with $T^{l^{\prime}} y=x$. Then by (3) in Lemma 2

$$
S_{m}(g(x))=\sum_{i \in J} g\left(T^{i+l^{\prime}}(y)\right)=\sum_{i \in J} g\left(T^{i}(x)\right)
$$

Now $T^{i}(x) \in T^{-(l-i)} F$, where $l-i \geq 0$, so by (iii) and (v)

$$
g\left(T^{i}(x)\right)=g^{l-i(\bmod N)}\left(T^{l}(x)\right)
$$

If $i$ runs through $J$ the indices $l-i \bmod N$ are all different and if $i \in J, i \pm K \notin J$. Thus the functions $g^{l-i(\bmod N)}(i \in J)$ are independent identically distributed and so are the functions $g \circ T^{i}(i \in J)$ with respect to $\left.\mu\right|_{T^{-l} F}$. Since

$$
\int_{T^{-l} F} g\left(T^{i}(x)\right) \mu(d x)=\int_{F} g^{l-i(\bmod N)} d \mu=0,
$$

it follows that

$$
\int_{T^{-\imath} F}\left|S_{m} g\right|^{2}=\int_{T^{-\imath} F} \sum_{i \in J} g^{2} \circ T^{i} d \mu=|J| \mu(F) .
$$

This shows the lemma, since we have

$$
\begin{aligned}
\left\|S_{m} g\right\|^{2} & \leq 4(K+1)^{2}\left(\varepsilon+L^{-1}\right)+(K+1)(L-1) N \mu(F) \\
& =K\left(1+K^{-1}+4 \frac{K+1}{K}(K+1)\left(\varepsilon+L^{-1}\right)\right) \\
\left\|S_{m} g\right\|^{2} & \geq(K-1)(L-1) N \mu(F) \\
& \geq(K-1) \frac{L-1}{L}(1-\varepsilon) \geq K\left(1-L^{-1}-\varepsilon-K^{-1}\right)
\end{aligned}
$$


and by the Central Limit Theorem with remainder

$$
\begin{aligned}
\mu(\{x & \left.\left.\in X \mid S_{m} g(x) / \sqrt{K} \leq t\right\}\right) \\
& \leq \mu(B)+\left.\sum_{l=N}^{L N-1} \mu\left(T^{-l} F\right) \mu\right|_{T^{-l} F}\left(\left\{x \in T^{-l} F \mid S_{m} g(x) / \sqrt{|J|} \leq \frac{K}{\sqrt{|J|}} t\right\}\right) \\
& \leq \varepsilon+L^{-1}+\sum_{l=N}^{L N-1} \mu\left(T^{-l} F\right)\left\{\Phi\left(\frac{K}{\sqrt{|J|}} t\right)+O\left(|J|^{-1 / 2}\right)\right\} \\
& =\Phi(t)+\zeta_{1}+O\left(K^{-1 / 2}\right), \\
\mu(\{x & \left.\left.\in X \mid S_{m} g(x) / \sqrt{K} \leq t\right\}\right) \\
\geq & \left.\sum_{l=N}^{L N-1} \mu\left(T^{-l} F\right) \mu\right|_{T^{-l} F}\left(\left\{x \in T^{-l} F \mid \frac{S_{m} g(x)}{\sqrt{|J|}} \leq \frac{K}{\sqrt{|J|}} t\right\}\right) \\
& =\sum_{l=N}^{L N-1} \mu\left(T^{-l} F\right)\left(\Phi\left(\frac{K}{\sqrt{|J|}} t\right)+O\left(|J|^{-1 / 2}\right)\right) \\
\geq & \left(1-L^{-1}\right)(1-\varepsilon)\left(\Phi(t)+\zeta_{2}+O\left(K^{-1 / 2}\right)\right) \\
= & \Phi(t)+\zeta_{3}+O\left(K^{-1 / 2}\right),
\end{aligned}
$$

where $\left|\varsigma_{3}\right|,\left|\xi_{1}\right| \rightarrow 0$ as $L, K \rightarrow \infty$ and $\varepsilon \rightarrow 0$.

We will now turn to the construction of special functions as we will need it below. We begin with

LEMMA 4. Let $F$ be an $(N L, \varepsilon)$-Rochlin set, $K<N, K$ odd and $N$ even. Let $\beta_{l}$ be a finite partition of $T^{-l} F(l=0,1, \ldots, L N-1)$. Then there exists a partition $\alpha$ of $F$ into $2^{N / 2}$ sets such that for every $A \in \alpha$ and $B \in \beta_{l}(0 \leq l<L N)$ $\mu\left(B \cap T^{-l} A\right)=2^{-N / 2} \mu(B)$.

ProOF. We will use Zorn's lemma as a convenience. Let $\Sigma$ be the family of all systems $\alpha=\left\{A_{1}, \ldots, A_{2^{N / 2}}\right\}$ where

(i) $A_{i} \cap A_{j}=\varnothing, A_{i} \subset F\left(1 \leq i \neq j \leq 2^{N / 2}\right)$.

(ii) If $B \in \beta_{l}$ then $\mu\left(B \cap T^{-l} A_{i}\right) \leq 2^{-N / 2} \mu(B)\left(1 \leq i \leq 2^{N / 2}\right)$.

If $\beta=\left\{B_{1}, \ldots, B_{2^{N / 2}}\right\}$ is another system then $A \leq B$ if and only if $A_{i} \subset B_{i}$, $i=1, \ldots, 2^{N / 2}$. Clearly, $\Sigma$ is nonempty and if $\Sigma_{0} \subset \Sigma$ is totally ordered, the system $\alpha=\left\{A_{1}, \ldots, A_{2^{N / 2}}\right\}$ defined by

$$
A_{i}=\bigcup_{B_{i} \in \alpha^{\prime} \in \Sigma_{0}} B_{i}
$$

belongs to $\Sigma$. Denote by $\alpha$ a maximal element in $\Sigma$. We claim that $\alpha$ is the partition we seek. For this it is sufficient to show that $\sum_{A \in \alpha} \mu(A)=\mu(F)$. Assume that this is not true. Let $c=\inf \mu\left(B \backslash \bigcup_{l=0}^{L N-1} T^{-l} \bigcup_{A \in \alpha} A\right)$ where the infimum extends over all $B \in \beta_{l}(l=0, \ldots, L N-1)$ for which the $\mu\left(B \backslash T^{-l} \bigcup_{A \in \alpha} A\right)>0$. Thus $c>0$ and therefore we can find a set $E \subset F \backslash \bigcup_{A \in \alpha} A$ with $0<\mu(E) \leq 2^{-N / 2} c$. Then for $B \in \beta_{l}$

$$
\mu\left(B \cap T^{-l} E\right) \leq \mu(E) \leq 2^{-N / 2} c
$$


if $\mu\left(B \backslash \bigcup_{A} T^{-l} A\right)>0$. Then define $\tilde{\alpha}$ by enlarging one set of $\alpha$ by $E$, and this contradicts the assumption since $\tilde{\alpha} \in \Sigma$. It is also clear that this argument may be made independent of the axiom of choice.

Now, let $\alpha$ be a partition as in the previous lemma. Write

$$
\alpha=\left\{A\left(\varepsilon_{0}, \ldots, \varepsilon_{N / 2-1}\right) \mid \varepsilon_{i} \in\{ \pm 1\}\right\} .
$$

Define functions $g^{2 l}(l=0, \ldots, N / 2-1)$ on $F$ by setting $g^{2 l}(x)=\varepsilon_{l}$ if $x \in$ $A\left(\varepsilon_{0}, \ldots, \varepsilon_{l}, \ldots, \varepsilon_{N / 2-1}\right)(l=0, \ldots, N / 2-1)$. Moreover we set $g^{2 l+K}(x)=-g^{2 l}$ $(l=0, \ldots, N / 2-1)$ where the indices are $\bmod N$ and

$(*) \quad g(x)= \begin{cases}g^{l}\left(T^{j N+l}(x)\right) & \text { if } x \in T^{-j N-l} F(0 \leq l<N, j=0, \ldots, L-1), \\ 0 & \text { if } x \notin \bigcup_{l=0}^{L N-1} T^{-l} F .\end{cases}$

LEMMA 5. The function $g$ defined in $(*)$ is special for $(F, N, K)$ and each of the functions $S_{m} g$, restricted to $T^{-l} F$, is independent of $\beta_{l}(l=N, \ldots, N L-1 ; 1 \leq$ $m \leq N)$ with respect to the measure $\left.\mu\right|_{T^{-\imath} F}$.

ProOF. That $g$ is special follows from the definition and Lemma 4: (i), (iv), and $(\mathrm{v})$ in the definition of a special function follow immediately from the above definitions of $g^{l}$ and $g$. We show (ii) and (iii).

(ii) Let $x \in F$. Then $g(x)=g^{0}(x)$ and for $\varepsilon \in\{ \pm 1\}$,

$$
\begin{aligned}
\mu(\{y \in F \mid g(y)=\varepsilon\}) & =\sum_{\varepsilon_{1}, \ldots, \varepsilon_{N / 2-1}} \mu\left(A\left(\varepsilon, \varepsilon_{1}, \ldots, \varepsilon_{N / 2-1}\right)\right) \\
& =2^{N / 2-1} 2^{-N / 2} \mu(F)=\frac{1}{2} \mu(F) .
\end{aligned}
$$

(iii) $\mu\left(g^{0}=\varepsilon_{0}, g^{2}=\varepsilon_{1}, \ldots, g^{N-2}=\varepsilon_{N / 2-1}\right)=\mu\left(A\left(\varepsilon_{0}, \ldots, \varepsilon_{N / 2-1}\right)\right)=$ $2^{N / 2-1} \mu(F)$ and $\mu\left(g^{2 l}=\varepsilon\right)=\frac{1}{2} \mu(F)$ (as in (ii)). Thus $g^{0}, g^{2}, \ldots, g^{N-2}$ are independent with respect to $\left.\mu\right|_{F}$.

Let us now show the additional independence statement in the lemma. Let $l \geq N$ and $m \leq N$ be fixed. Fix an atom $B \in \beta_{l}$ and let $A=\left\{x \in T^{-l} F \mid g\left(T^{j}(x)\right)=\right.$ $\left.\varepsilon_{j}, 0 \leq \bar{j}<m\right\}$, where a priori $\varepsilon_{j} \in\{-1,0,1\}$. But for $x \in T^{-l} F g\left(T^{j}(x)\right)=0$ never happens $(l-j \geq 0$ for all $j<m \leq N)$, so we take $\varepsilon_{j} \in\{ \pm 1\}$. We have seen earlier that for $x \in T^{-l} F g\left(T^{j}(x)\right)=g^{l-j(\bmod N)}\left(T^{l}(x)\right)$. It follows that either $A=\varnothing$ (if the $\varepsilon_{j}$ do not match, condition (iv)) or $A$ is a finite union of sets $T^{-l} A\left(\eta_{1}, \ldots, \eta_{N / 2-1}\right)$ with $\eta_{i} \in\{ \pm 1\}$ (depending on $m$ and $l$ ). It follows from Lemma 4 that

$$
\begin{aligned}
\left.\mu\right|_{T^{-l} F}(B \cap A) & =\left.\mu\right|_{T^{-l} F}\left(B \cap U T^{-l} A\left(\eta_{0}, \ldots, \eta_{N / 2-1}\right)\right) \\
& =\mu(F)^{-1} \sum \mu\left(B \cap T^{-l} A\left(\eta_{0}, \ldots, \eta_{N / 2-1}\right)\right) \\
& =\mu(F)^{-1} \sum 2^{-N / 2} \mu(B)=[\mu(A) / \mu(F)][\mu(B) / \mu(F)]
\end{aligned}
$$

since

$$
\mu(A)=\sum \mu\left(A\left(\eta_{0} \eta_{1} \cdots \eta_{N / 2-1}\right)\right)=\sum_{A\left(\eta_{0}, \ldots, \eta_{N / 2-1}\right) \subset A} 2^{-N / 2} \mu(F)
$$

After these preparations we are ready to define the functions $f: X \rightarrow \mathbf{R}$ for which we will show the CLT property below. We start with a sequence $\varepsilon_{n} \searrow 0$, a sequence 
$K_{n} \nearrow \infty$ of odd integers, a sequence $N_{n} \nearrow \infty$ of even integers, and a sequence $L_{n} \nearrow \infty$ of integers $(n \geq 1)$ satisfying (c) below and
(a) $K_{n}<N_{n-1}$,
(b) $K_{n}\left(L_{n}^{-1}+\varepsilon_{n}\right) \rightarrow 0(n \rightarrow \infty)$.

Let $F_{n}(n \geq 1)$ be $\left(N_{n} L_{n}, \varepsilon_{n}\right)$-Rochlin sets and let $g_{n}$ be special functions for $\left(F_{n}, N_{n}, K_{n}\right)$ such that for each $m=1, \ldots, N_{n}, S_{m} g_{n}$ is independent on $T^{-l} F_{n}$ $(l=N, \ldots, L N-1)$ of the partition generated by $\left\{T^{i} g_{n-1} \mid 0 \leq i<N_{n}\right\}$ (w.r.t. $\left.\left.\mu\right|_{T^{-\imath} F_{n}}\right)$. By Lemma 5, this is possible. Finally, let $a_{n} \searrow 0(n \rightarrow \infty)$ be a sequence of positive real numbers, such that

$$
\begin{cases}\sum a_{2}^{2}<\infty & \\ a_{n}^{-1} \sum_{j>n} a_{j} \rightarrow 0 & \text { as } n \rightarrow \infty \\ a_{n}^{-1} K_{n}^{-1 / 2} \sum_{j<n} a_{j} K_{j} \rightarrow 0 & \text { as } n \rightarrow \infty .\end{cases}
$$

Then we define $f=\sum_{n \geq 1} a_{n} g_{n}$. It is left to show that $f$ satisfies the CLT. Given $m \geq 1$ define $n_{0}=n_{0}(m)=\sup \left\{n \mid K_{n} \leq m\right\}$.

LEMMA 6. We have

$$
\begin{aligned}
(1-\delta)\left(K_{n_{0}} a_{n_{0}}^{2}+m a_{n_{0}+1}^{2}\right) & \leq\left\|S_{m}\left(a_{n_{0}} g_{n_{0}}+a_{n_{0}+1} g_{n_{0}+1}\right)\right\|^{2} \\
& \leq(1+\delta)\left(K_{n_{0}} a_{n_{0}}^{2}+m a_{n_{0}+1}^{2}\right)
\end{aligned}
$$

where $\delta \rightarrow 0$ as $m \rightarrow \infty$.

$$
\left(K_{n_{0}} a_{n_{0}}^{2}+m a_{n_{0}+1}^{2}\right)^{-1}\left\|\sum_{\substack{n \neq n_{0} \\ n \neq n_{0}+1}} S_{m}\left(a_{n} g_{n}\right)\right\|^{2} \rightarrow 0 \text { as } m \rightarrow \infty .
$$

PROOF. (1) Since $m<K_{n+1}<N_{n}, S_{m}\left(a_{n_{0}+1} g_{n_{0}+1}\right)$ is independent of $S_{m}\left(a_{n_{0}} g_{n_{0}}\right)$ on each level $T^{-l} F_{n_{0}+1}\left(N_{n_{0}+1} \leq l<L_{n_{0}+1} N_{n_{0}+1}\right)$ (w.r.t. $\left.\mu\right|_{T^{-l} F_{n_{0}+1}}$ ). Thus if $G=\bigcup_{l=N_{n_{0}+1}}^{L_{n_{0}+1} N_{n_{0}+1}-1} T^{-l} F, B=G^{c}$, by Lemma 3

$$
\begin{aligned}
\| S_{m}( & \left.a_{n_{0}} g_{n_{0}}+a_{n_{0}+1} g_{n_{0}+1}\right) \|^{2} \\
\leq & 2 \int_{B}\left(S_{m}\left(a_{n_{0}} g_{n_{0}}\right)\right)^{2} d \mu+2 \int_{B}\left(S_{m}\left(a_{n_{0}+1} g_{n_{0}+1}\right)\right)^{2} d \mu \\
& +\sum_{l=N_{n_{0}+1}}^{L_{n_{0}+1} N_{n_{0}+1}-1} \int_{T^{-l} F_{n_{0}+1}}\left(S_{m}\left(a_{n_{0}} g_{n_{0}}\right)\right)^{2} \\
& +\int_{T^{-l} F_{n_{0}+1}}\left(S_{m}\left(a_{n_{0}+1} g_{n_{0}+1}\right)\right)^{2} d \mu \\
\leq & 8\left[a_{n_{0}}^{2}\left(K_{n_{0}+1}\right)^{2}+a_{n_{0}+1}^{2} m^{2}\right]\left(\varepsilon_{n_{0}+1}+L_{n_{0}+1}^{-1}\right) \\
& +\left\|S_{m}\left(a_{n_{0}} g_{n_{0}}\right)\right\|^{2}+\left\|S_{m}\left(a_{n_{0}+1} g_{n_{0}+1}\right)\right\|^{2} \\
= & {\left[a_{n_{0}}^{2} K_{n_{0}}+a_{n_{0}+1}^{2} m\right]\left(1+\delta^{\prime}\right) }
\end{aligned}
$$


where $\delta^{\prime} \rightarrow 0$ as $m \rightarrow \infty$ by our general assumptions. By a similar argument one obtains

$$
\begin{aligned}
\left\|S_{m}\left(a_{n_{0}} g_{n_{0}}+a_{n_{0}+1} g_{n_{0}+1}\right)\right\|^{2} \geq & \left\|S_{m}\left(a_{n_{0}} g_{n_{0}}\right)\right\|^{2}+\left\|S_{m}\left(a_{n_{0}+1} g_{n_{0}+1}\right)\right\|^{2} \\
& -4\left[a_{n_{0}}^{2}\left(K_{n_{0}+1}\right)^{2}+a_{n_{0}+1}^{2} m^{2}\right] \\
= & {\left[a_{n_{0}}^{2} K_{n_{0}}+a_{n_{0}+1}^{2} m\right]\left(1-\delta^{\prime}\right) }
\end{aligned}
$$

where $\delta^{\prime} \rightarrow 0$.

(2) Using Lemma 3 again we have

$$
\left\|\sum_{\substack{n \neq n_{0} \\ n \neq n_{0}+1}} S_{m}\left(a_{n} g_{n}\right)\right\| \leq \sum_{\substack{n \neq n_{0} \\ n \neq n_{0}+1}}\left\|S_{m}\left(a_{n} g_{n}\right)\right\| \leq \sum_{n<n_{0}} 2\left(K_{n}+1\right) a_{n}+\sum_{n \geq n_{0}+2} m a_{n}
$$

and (2) follows from (c).

LEMMA 7. We have

$$
\mu\left(\left\{x \in X \mid \frac{S_{m}\left(a_{n_{0}} g_{n_{0}}+a_{n_{0}+1} g_{n_{0}+1}\right)(x)}{\left(K_{n_{0}} a_{n_{0}}^{2}+m a_{n_{0}+1}^{2}\right)^{1 / 2}} \leq t\right\}\right) \rightarrow \phi(t)
$$

as $m \rightarrow \infty$.

PROOF. As before, let $g=\bigcup_{l=N_{n_{0}+1}}^{L_{n_{0}+1} N_{n_{0}+1}-1} T^{-l} F_{n_{0}+1}$ and $B=G^{c}$. Since $S_{m}\left(a_{n_{0}+1} g_{n_{0}+1}\right)$ is independent of $S_{m}\left(a_{n_{0}} g_{n_{0}}\right)$ on $T^{-l} F_{n_{0}+1}$ with respect to $\left.\mu\right|_{T^{-l} F_{n_{0}+1}}\left(N_{n_{0}+1} \leq l<L_{n_{0}+1} N_{n_{0}+1}\right)$ we have (using Fourier transforms):

$$
\begin{aligned}
\int_{G} \exp & \left(i t \frac{S_{m}\left(a_{n_{0}} g_{n_{0}}+a_{n_{0}+1} g_{n_{0}+1}\right)}{\left(a_{n_{0}}^{2} K_{n_{0}}+a_{n_{0}+1}^{2} m\right)^{1 / 2}}\right) d \mu \\
= & \sum_{l=N_{n_{0}+1}}^{L_{n_{0}+1} N_{n_{0}+1}} \mu\left(T^{-l} F_{n_{0}+1}\right) \\
& \left.\cdot \int \exp \left(i t \frac{S_{m}\left(a_{n_{0}} g_{n_{0}}+a_{n_{0}+1} g_{n_{0}+1}\right)}{\left(a_{n_{0}}^{2} K_{n_{0}}+a_{n_{0}+1}^{2} m\right)^{1 / 2}}\right) d \mu\right|_{T^{-\varphi} F_{n_{0}+1}} \\
= & \sum_{l=N_{n_{0}+1}}^{L_{n_{0}+1} N_{n_{0}+1}} \mu\left(T^{-l} F_{n_{0}+1}\right) \\
& \left.\cdot \int \exp \left(i t \frac{a_{n_{0}} K_{n_{0}}^{1 / 2}}{\left(a_{n_{0}}^{2} K_{n_{0}}+a_{n_{0}+1}^{2} m\right)^{1 / 2}} \frac{S_{m}\left(a_{n_{0}} g_{n_{0}}\right)}{a_{n_{0}} K_{n_{0}}^{1 / 2}}\right) d \mu\right|_{T^{-l} F_{n_{0}+1}} \\
& \cdot\left(\exp \left(\frac{1}{2} \frac{a_{n_{0}+1}^{2} m t^{2}}{a_{n_{0}}^{2} K_{n_{0}}+a_{n_{0}+1}^{2} m}\right)+\eta_{m}\right)
\end{aligned}
$$


where $\eta_{m} \rightarrow 0$ as $m \rightarrow \infty$, because $S_{m}\left(a_{n_{0}+1} g_{n_{0}+1}\right) / a_{n_{0}+1} m^{1 / 2}$ converges to the standard normal distribution. Hence the last expression equals

$$
\begin{gathered}
\int_{G} \exp \left(i t \frac{a_{n_{0}} K_{n_{0}}^{1 / 2}}{\left(a_{n_{0}}^{2} K_{n_{0}}+a_{n_{0}+1}^{2} m\right)^{1 / 2}} \frac{S_{m}\left(a_{n_{0}} g_{n_{0}}\right)}{a_{n_{0}} K_{n_{0}}^{1 / 2}}\right) d \mu \\
\cdot\left(\exp \left(-\frac{1}{2} \frac{a_{n_{0}+1}^{2} m t^{2}}{a_{n_{0}}^{2} K_{n_{0}}+a_{n_{0}+1}^{2} m}\right)+\eta_{m}\right)
\end{gathered}
$$

and this converges to $\exp -\frac{1}{2} t^{2}$, since $S_{m}\left(a_{n_{0}} g_{n_{0}}\right) / a_{n_{0}} K_{n_{0}}^{1 / 2}$ converges to the standard normal distribution and since $\mu(G) \rightarrow 1$. Lemmas 6 and 7 now imply that $f$ satisfies the CLT. Lemma 6 shows that for a given $m$ only the contributions of $a_{n} g_{n}+a_{n+1} g_{n+1}\left(n=n_{0}(m)\right)$ determine the limit and by Lemma 7 this limit exists and is the standard normal distribution. The details are carried out as in the proof of Theorem 1a for the irrational rotation.

\section{REFERENCES}

1. R. N. Bhattacharya, On the functional central limit theorem and the law of the iterated logarithm for Markov processes, Z. Wahrsch. Verw. Gebiete 60 (1982), 185-201.

2. P. Billingsley, Convergence of probability measures, Wiley, New York, 1968.

3. M. Denker and M. Keane, Almost topological dynamical systems, Israel J. Math. 34 (1979), 139-160.

4. M. Denker and W. Philipp, Approximation by Brownian motion for Gibbs measures and flows under a function, Ergodic Theory Dynamical Systems 4 (1984), 541-552.

5. D. Dürr and S. Goldstein, Remarks and the central limit theorem for weakly dependent sequences, Preprint, 1985.

6. M. I. Gordin and B. A. Lifsic, The central limit theorem for stationary Markov processes, Soviet Math. Dokl. 19 (1978), 392-394.

7. F. Hofbauer and G. Keller, Ergodic properties of invariant measures for piecewise monotonic transformations, Math. Z. 180 (1982), 119-140.

8. I. A. Ibragimov, Some limit theorems for stationary processes, Theory Probab. Appl. 7 (1962), 349-382.

9. G. Maruyama, Nonlinear functions of Gaussian stationary processes and their applications, (Proc. Third Japan-U.S.S.R. Sympos. on Probability), Lecture Notes in Math., vol. 550, Springer, 1975, pp. 375-378.

10. C. M. Newman and A. L. Wright, An invariance principle for certain dependent sequences, Ann. Probab. 9 (1981), 671-675.

11. W. Philipp and W. Stout, Almost sure invariance principles for partial sums of weakly dependent random variables, Mem. Amer. Math. Soc. No. 161, 1975.

12. M. Ratner, The central limit theorem for geodesic flows on $n$-dimensional manifolds of negative curvature, Israel J. Math. 16 (1973), 181-197.

13. A. Rényi, Wahrscheinlichkeitrechnung, VEB Deutscher Verlag Wiss., Berlin, 1962.

14. M. Rosenblatt, $A$ central limit theorem and a strong mixing condition, Proc. Nat. Acad. Sci. U.S.A. 42 (1956), 43-47.

15. A. Rothstein, Personal communication, 1984.

16. R. Salem and A. Zygmund, On lacunary trigonometric series, Proc. Nat. Acad. Sci. U.S.A. 33 (1947), 333-338; II ibid. 34 (1948), 54-62.

\footnotetext{
Department of Mathematics, Oregon State University, Corvallis, Oregon 97331

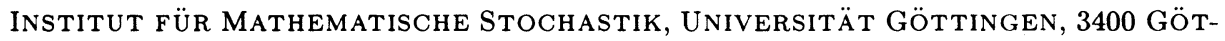
TINGEN, FEDERAL REPUBLIC OF GERMANY
} 